\title{
Rhétorique et herméneutique : imprécations raciniennes
}

Delphine Denis

\section{(2) OpenEdition \\ 12 Journals}

Édition électronique

URL : http://journals.openedition.org/rhetorique/101

DOI : 10.4000/rhetorique.101

ISSN : 2270-6909

Éditeur

UGA Éditions/Université Grenoble Alpes

Édition imprimée

ISBN : 978-2-84310-255-4

Référence électronique

Delphine Denis, «Rhétorique et herméneutique : imprécations raciniennes », Exercices de rhétorique [En ligne], 1 | 2013, mis en ligne le 06 novembre 2013, consulté le 12 septembre 2020. URL : http:// journals.openedition.org/rhetorique/101; DOI : https://doi.org/10.4000/rhetorique.101

Ce document a été généré automatiquement le 12 septembre 2020.

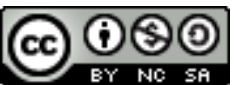

Les contenus de la revue Exercices de rhétorique sont mis à disposition selon les termes de la Licence Creative Commons Attribution - Pas d'Utilisation Commerciale - Partage dans les Mêmes Conditions 4.0 International. 


\title{
Rhétorique et herméneutique : imprécations raciniennes*
}

\author{
Delphine Denis
}

1 Si l'ars rhetorica est bien, à l'origine, conçue comme un ensemble ordonné de techniques visant à élaborer et produire un discours persuasif, c'est-à-dire efficace, ce savoir-faire s'est très vite accompagné d'un " savoir-lire ${ }^{1}$ ». L'âge classique non seulement en hérite, mais encore assume explicitement cette inflexion, nourrie de la pratique du commentaire savant ou pédagogique. À une "première rhétorique " succède ainsi sa seconde version, selon les termes mêmes d'un obscur et prolixe professeur actif au milieu du XVII ${ }^{\mathrm{e}}$ siècle :

Par l'adresse de la composition, qui est la première fonction et le premier usage de l'une et de l'autre Éloquence, on a su se donner des règles pour se conduire judicieusement dans la méditation de quelque sujet, et pour le traiter si savamment, qu'aucune des plus belles pensées qui le regardent ne nous puissent échapper [...]. Par l'industrie de la dissection ou critique, qui est la seconde démarche de l'Art du Discours, et tout à fait opposée à la précédente, on s'est acquis la facilité d'être excellent Orateur, sans génie, sans peine, sans étude, sans l'artifice de la préparation, et sans le secours de la lecture.

Il semble que ces deux actions de l'Art oratoire comprennent tous les usages de cette divine Faculté ${ }^{2}[\ldots]$.

Pour que ce geste critique nous paraisse aujourd'hui légitime, c'est bien que ce déplacement, historiquement attesté, a réussi. La présente étude s'inscrit dans une telle démarche, jalonnée par de nombreux travaux : elle s'attachera à restituer à une notion rhétorique (l'imprécation) les cadres de sa théorisation, puis sa mise en œuvre textuelle au sein de deux tirades extraites de Britannicus et d'Athalie de Racine ${ }^{3}$, avant d'interroger le possible rendement herméneutique que cette confrontation entre histoire profane et histoire sacrée est susceptible de livrer. Au sein de l'immense matière rhétorique que le poète met en œuvre ${ }^{4}$, l'imprécation, on le verra, a partie liée avec la question du sublime, tout en offrant une représentation naturelle - c'est-à-dire motivée et vraisemblable - des passions dramatiques qu'il s'agit de représenter, pour susciter l'émotion nécessaire du spectateur : «les "fureurs", les "imprécations", voilà 
qui, dans Racine, paraît le plus humain ", écrivait François Mauriac dans sa Vie de Jean Racine ${ }^{5}$.

1.

3 Si nous savons, intuitivement, ce qu'est une imprécation, ce souhait de malheur proféré à l'encontre de quelqu'un, et pouvons donc en repérer les séquences textuelles, c'est d'abord dans la mesure où ces malédictions relèvent d'un acte de langage culturellement - voire universellement - partagé. Peut-être suffirait-il de s'en tenir à cette approche empirique. Mais ce serait faire peu de cas des analyses proposées en ces temps anciens, soucieux de discuter la tradition qu'ils relaient.

4 L'enquête philologique s'impose alors en préalable. Le répertoire de Lausberg' ${ }^{6}$, qui reste une référence pour l'Antiquité, n'en propose pourtant aucune entrée, que ce soit sous imprecatio, deprecatio, execratio, maledictio, juramentum, etc. Est-ce à dire que la notion est inconnue des anciens traités de rhétorique, alors même que la pratique s'en repère aisément à l'âge classique, comme le montrent les commentaires de l'époque? Leur consultation, qu'il s'agisse du domaine profane ou des textes sacrés, livre en effet une abondante moisson ${ }^{7}$. Sont ainsi allégués, entre autres, des passages empruntés aux Catilinaires de Cicéron ${ }^{8}$, à ses Philippiques $^{9}$ et ses Lettres familières ${ }^{10}$, au chant IV de L'Énéide de Virgile où Didon abandonnée s'adresse en fureur au héros de l'épopée ${ }^{11}$, à l'Ancien Testament ou aux Pères de l'Église ${ }^{12}$. Nous sommes donc bien en présence d'une notion pertinente pour l'analyse des textes, et qui fait parfois même l'objet de brèves définitions. Dans le traité de Du Cygne (1670), au chapitre IV, c'est au titre des vingt-cinq figures de pensée que l'on rencontre la déprécation et l'exécration, aux côtés de l'interrogation, de l'apostrophe, de la prosopopée, de la concession ou encore de l'ironie : autant de figures qui relèvent d'actes de langage que l'auteur jugent les mieux aptes à émouvoir les passions (aptiores ad concitendos affectus). À la fin du XVIII ${ }^{\mathrm{e}}$ siècle, le manuel de Nicolas Hurtaut classe lui aussi l'imprécation ou exécration dans la même catégorie des figures de pensée propres à émouvoir ( $a d$ movendum). Lorsque l'étude en est abordée, l'auteur illustre son propos par des exemples de nouveau empruntés à Cicéron ou à Virgile. Mais il y ajoute deux extraits de tragédies désormais "classiques »: c'est Corneille qui lui en fournit la matière, avec la tirade de Camille dans Horace $(\mathrm{IV}, 5)$ et celle de Cléopâtre dans Rodogune $(\mathrm{V}, 4)$.

5 De fait, les deux grands traités de poétique dramatique à l'âge classique ${ }^{13}$ font place à l'imprécation dans leur examen des formes de discours particulièrement adaptées à l'expression théâtrale. Cette "parole agissante ", comme ils la définissent ${ }^{14}$, est à mettre en relation avec l'émotion pathétique, de même qu'en retour « la scène est à bien parler le Trône des grands mouvements ${ }^{15}$ ». Dans le traité de La Mesnardière, c'est au chapitre $\mathrm{X}$, consacré au langage, quatrième partie de la tragédie selon l'auteur, qu'est abordée la question des «sentiments passionnés» (opposés aux «sentiments indifférents ", selon les deux modalités qui régissent les "pensées du poème » dramatique). Au nombre de quatre (douleur, colère, amour et jalousie), leur combinatoire sous le chef de l'imprécation est bien repérée, même si le terme ne figure qu'incidemment dans le traité. Douleur et colère mêlées sont qualifiées en ces termes :

J'appelle mouvement de rage, cette passion tumultueuse dont un esprit est agité dans les malheurs insupportables qui surmontent sa patience ${ }^{16}$. 
6 Puisque « la colère et la fureur parlent d'un ton impétueux, foudroyant et précipité ${ }^{17}$ ", cette "passion tumultueuse » appelle "pour son caractère les gémissements, le tumulte, les clameurs et les tempêtes ${ }^{18} »$. Mais les exemples allégués par l'auteur ne permettent pas de distinguer, au sein de ces séquences passionnées, le régime singulier de l'imprécation : si l'on y retrouve l'épisode virgilien de Didon abandonnée, désigné comme "frénésie", celui-ci s'insère dans une série allusive où sont également convoqués la fureur d'Ajax, « le désespoir de Thisbé après la mort de Pyrame, et la rage de Prométhée attaché sur le mont Caucase ${ }^{19} »$.

7 L'analyse se précise quelque quinze ans plus tard, dans La Pratique du théâtre de l'abbé d'Aubignac. Celui-ci repère quatre sortes de discours propres au genre dramatique. Dès le deuxième chapitre, consacré aux narrations, l'imprécation est signalée comme l'un des ressorts du pathétique :

Les Narrations pathétiques sont toujours les plus belles, et celles qu'on peut nommer seules dignes du Théâtre, lorsqu'elles sont soutenues d'une exagération raisonnable, et de toutes les circonstances importantes d'une histoire; qu'elles sont mêlées d'étonnement, d'imprécations, de crainte, et d'autres emportements d'esprit selon les diverses impressions qui doivent naître du Récit ${ }^{20}$.

Dans le chapitre VII, « Discours pathétique, ou Des passions et mouvements d'esprit ${ }^{21}$ ", l'imprécation est tenue pour l'un des «beaux endroits » attendus des spectateurs, dont il s'agit d' «enlever l'esprit ${ }^{22}$ » par un mouvement croissant d'émotion : cette dynamique ressortit tant à l'inventio rhétorique qu'à la dispositio qui en ménage les paliers, et à l'elocutio qui en soutient l'expression. C'est enfin au chapitre suivant, qui s'arrête sur les figures de pensée ${ }^{23}$, qu'intervient l'imprécation, l'une des plus « impétueuses ${ }^{24}$ ", des plus " grandes et sérieuses aussi ${ }^{25}$ ». De cet ensemble, certaines s'avèrent «plus propres au Théâtre que les autres ${ }^{26} »:$ dans l'énumération que fournit l'auteur, et qui regroupe l'apostrophe, «fort éclatante, quand elle est bien placée et bien conduite ${ }^{27}$ ", la prosopopée, l'ironie, l'exclamation, l'hyperbole et l'interrogation ${ }^{28}$, il ressort qu' « entre toutes, l'imprécation sera jugée certainement théâtrale ${ }^{29}$ ».

9 De cette rapide enquête, à laquelle il faudrait joindre l'analyse proposée par les commentateurs des textes vétérotestamentaires, où l'imprécation est constamment relevée, il suffira de retenir pour notre propos que cette séquence :

- est repérée, c'est-à-dire lue et découpée comme telle par les traités et commentaires de l'âge classique ;

- peut être mise en série avec les ressources de la colère et de la douleur, à l'instar des « figures de pensée » qui relèvent d'une économie globale du discours. Elle intéresse donc à ce titre l'inventio (pour en motiver ou en retrouver la mise en œuvre), la dispositio (pour en organiser le crescendo pathétique dont elle est l'un des temps forts, sans pour autant se confondre avec le dénouement qui peut toujours en renverser l'effet) et l'elocutio (qui en portera l'expression) - à charge pour les acteurs de la relayer dans l'ordre de la memoria et de l'actio ;

- enfin, qu'elle est tout particulièrement bienvenue sur la scène tragique, dont elle accentue le pathétique, tout en laissant ouverte la dimension "agissante » de sa profération. Seule en effet, l'Histoire - profane ou sacrée - en aura validé l'efficacité performative.

10 Aussi est-il nécessaire d'opérer une seconde contextualisation, en revenant enfin à notre proposition de lecture des deux tirades raciniennes. La première en effet appartient à l'histoire romaine, l'autre est prise d'un sujet biblique. Leur confrontation, comme on le verra, est aussi légitime que problématique. 
2.

11 Ces deux tirades prennent place dans une même situation dramatique, où les deux phases d'inventio et de dispositio se voient, comme souvent, étroitement imbriquées. L'une et l'autre en effet interviennent au dernier acte de la pièce, dans cette position de dénouement qui achève la mise en intrigue, et dont la logique commandait la construction de l'œuvre. Il rend possible, rétrospectivement, une interprétation d'ensemble : celle-ci tout à la fois marque la clôture dans l'ordre du déroulement des événements, et ouvre sur des conséquences imaginables, voire prévisibles.

Dans les deux cas, également, le personnel dramatique met aux prises les mères et les fils (ou petit-fils, s'agissant de Joas). La défaite politique des mères est patente, chassées du pouvoir par leurs rejetons: Néron, qui a perpétré son premier crime, affronte directement les décisions d'Agrippine (et menace à terme sa place au sein de la Cour, voire sa survie) ; Joas vient d'être déclaré roi légitime au prix d'un complot dont Athalie et son prêtre Mathan sont les victimes directes. Cette victoire des fils contre les mères, matricide symbolique, en annonce la fin prochaine. En se tournant contre leur propre sang, dont les liens originels seront rompus dans et par le même sang, la malédiction des mères illustre la parfaite convenance de la fable au genre de la tragédie: cette violence au sein des alliances, qui était déjà pour Aristote la meilleure voie pour provoquer terreur et pitié $e^{30}$, et que Corneille commente dans son traité De la Tragédie ${ }^{31}$, s'exerce ici exemplairement. Un même motif parcourt ainsi les deux pièces : celui du "sang détestable ${ }^{32}$ ", de la race maudite, du lien filial corrompu. Qu'il relève d'une tradition profane (avec la fable des Atrides pour les Grecs ou celle de la fondation de Rome, marquée par l'assassinat de Rémus par son frère jumeau ${ }^{33}$ ) ou chrétienne (le meurtre originel d'Abel par Caï ${ }^{34}$ ), Racine y trouve une ample matière en résonance avec ses propres préoccupations spirituelles, nourries de la lecture augustinienne à Port-Royal ${ }^{35}$. Aussi les deux sujets des œuvres ici retenues s'inscrivent-ils dans une chaîne de crimes inauguraux. Leur dénouement, loin d'en borner le terme, ne constitue à cet égard qu'un prologue pour de futurs forfaits que connaissent déjà spectateurs et lecteurs. Les mères le savent, qui en prédisent la suite dans nos deux tirades : «Par des faits glorieux tu te vas signaler », déclare Agrippine à Néron ; «Qu'il règne donc, ce Fils, ton soin et ton ouvrage, / Et que pour signaler son empire nouveau / On lui fasse en mon sein enfoncer ce couteau ${ }^{36}[. .]$.$» , tel est le terrible vœu que formule Athalie. Au$ cœur de cette interrogation cruciale sur la filiation et l'ordre du monde, la légitimité trahie ou reconnue passe par l'avènement d'un nom propre, qu'il s'agit de faire advenir : pour Néron, voir enfin dans le fils d'Agrippine le nouveau César-Auguste ${ }^{37}$, et pour Joas, recouvrer derrière la fausse identité de l'enfant sauvé Éliacin, celle de l'héritier du roi David, garant de l'Alliance entre Dieu et le peuple élu.

Ainsi portées par un sujet tragique, aux effets pathétiques surdéterminés, les imprécations raciniennes ne surgissent-elles pas sans avoir été longuement préparées par le dramaturge. Leur véhémence verbale, bienséante dans sa dignité d'expression et dans sa convenance aux caractères représentés, est le pendant rhétorique d'une violence physique que le théâtre ne souffre plus sur scène.

Répertoriée comme on l'a vu au rang des «figures de pensée », l'imprécation partage avec quelques autres (par exemple, l'apostrophe, l'exclamation ou l'interrogation) le statut d'un acte de langage à part entière ${ }^{38}$. Celui-ci se traduit par la présence de verbes 
introducteurs de discours indirect qui en explicitent la nature (souhaiter, vouloir, espérer et plus encore prévoir ${ }^{39}$ ), par le recours aux modalités optative ${ }^{40}$ ou volitive ${ }^{41}$, enfin par la visée prospective d'une temporalité tournée vers un avenir proche, au delà du dénouement représentét ${ }^{42}$. Engageant les passions violentes de la colère et de la douleur, elle déploie dans les deux tirades une commune isotopie de la haine et du sang maudit, ainsi qu'une expression paroxystique privilégiant toutes les formes du haut degré (indéfinis de la totalité ${ }^{43}$, déterminants intensifs ${ }^{44}$, hyperboles ${ }^{45}$ ), dont l'effet pathétique est ici saisissant. Il est mis au service d'un sublime "noir", attaché aux paroles testamentaires de ces mères qui les veulent et les croient dotées d'une efficacité post mortem : "en mourant ${ }^{46}$ ", elles entendent précipiter dans leur chute le fils maudit, digne rejeton d'une race exécrable. L'analyse que propose Michel Deguy du " granddire » selon Longin trouve ici toute sa pertinence :

La condition mortelle et le moment du périr sont en jeu avec le sublime. Est sublime le rassemblement, le sursaut, qui équilibre le mourir en une parole - dont une typologie devrait démultiplier le genre : bénédiction ou malédiction qui retombe sur les vivants et rebondissant sur la tombe, hyperbole de l'épitaphe improvisée ; défi, supplication, salut, serment, énigme, exécration, sarcasme, ou ce que les Latins appellent de-votio...

Il y a un rapport du sublime au testamentaire. Les mots sublimes sont les mots de la $\mathrm{fin}^{47}$.

15 Ces «mots de la fin », qu'enchâssent adverbes et présentatifs" ${ }^{48}$, soutenus par le jeu de l'hypotypose $\mathrm{e}^{49}$ et de l'ironie ${ }^{50}$ donnent à l'ensemble de la tirade sa portée pathétique. Mais au delà de cet effet attendu, ils prennent une dimension mémorielle : tout se passe comme si ces deux malédictions maternelles, par le sur-marquage de leur caractère solennel, voire formulaire, acquéraient d'emblée le statut de citation, qu'en réalité seule l'épreuve des temps à venir peut leur conférer.

\section{3.}

Or, c'est bien les leçons de l'Histoire que ces deux tirades viennent relayer et interroger. Leurs indéniables parentés structurelles et stylistiques, que l'on espère avoir mis en lumière, ne doivent pas masquer la divergence d'interprétations auxquelles invite le texte de Racine. L'approche rhétorique conduite jusqu'ici nous semble devoir être prolongée pour déboucher, sans se trahir, sur une herméneutique.

L'examen de la situation de communication des deux tirades, derrière l'apparence d'une commune scène d'affrontement, révèle une radicale dissymétrie dans le fonctionnement de l'échange. Si dans Britannicus en effet, il s'agit bien d'une scène d' agôn, justifiant l'adresse directe d'Agrippine à Néron (impératifs, apostrophe, marques diverses de l'énonciation à la P2), la tirade d'Athalie opère une sortie hors du dialogue dramatique. Ce débrayage énonciatif, cependant, n'est que partiel : en choisissant, au sein d'une séquence peuplée de nombreux personnages (dont Joas, le grand-prêtre Joad qui l'a protégé jusque là sous le nom d'Éliacin, et sa femme Josabeth) directement intéressés par les événements qui se précipitent, de représenter Athalie tournée vers le "Dieu des Juifs», Racine conserve toute la tension pathétique qui tient à l'interpellation directe. Mais ce "Dieu caché » le reste aux yeux de tous, personnages et spectateurs. En le défiant, Athalie le constitue en seul véritable interlocuteur, par delà ses porte-parole ou ses officiants: elle entérine ainsi la victoire ultime du Dieu de colère (« impitoyable Dieu ») et de sa race élue (« David, David triomphe. Achab seul est 
détruit »). Du même coup, la malédiction proférée à l'encontre de son petit-fils Joas ne peut l'être qu'indirectement, par énallage. Au lieu des deux personnes du discours adressé, l'imprécation emprunte le détour de la seule P3 («Voici ce qu'en mourant lui souhaite sa Mère ", v. 1783), qui s'accompagne, dans le dernier vers, de l'infinitif, forme verbale non embrayée ("Et venger Athalie»). De la même logique procèdent les désignations métonymiques (" un sang, que je déteste ", v.1772) ou périphrastiques de l'enfant reconnu et enfin nommé (« Oui, c'est Joas », v. 1769) : « un enfant » (v. 1777), « ce Fils, ton soin, et ton ouvrage » (v. 1780), « de David l'héritier détestable » (v. 1788).

Prononcées dans ces régimes distincts de l'énonciation, les deux imprécations relèvent ainsi de logiques différentes. Dans Britannicus, Agrippine peut prédire, sur la foi du vraisemblable éthique d'origine aristotélicienne - celui du « caractère » de Néron dont la cohérence à venir est présumée par ses premiers forfaits -, le " monstre naissant " que Racine entendait représenter ${ }^{51}$ : les impératifs en anaphore soulignés par les coupes métriques («Poursuis », v. 1692 et 1694) signalent cette continuité ( Je prévois que tes coups viendront jusqu'à ta Mère », v. 1696 ; «Voilà ce que mon cœur se présage de toi », v. 1713). Il n'en va pas ainsi d'Athalie, qui n'avait pas su reconnaitre, dans tous les sens du terme, et malgré tous les présages, l'enfant cru assassiné. L'imprécation s'exerce comme une forme d'inversion blasphématoire, diabolique, de toutes les prières qui scandent la pièce. En alléguant contre elle-même et son propre sang la malédiction divine proférée envers la race d'Achab, Athalie renvoie le Créateur à son œuvre, et confirme ainsi la toute-puissance du Verbe : «Fidèle au sang d'Achab, qu'il a reçu de moi, / Conforme à son Aïeul, à son Père semblable » (v. 1786-1787), Joas se voit ainsi promis à une destinée d'apostasie («indocile à ton joug, fatigué de ta Loi », v. 1785) qui le conduira à " abolir » et " profaner » ces mêmes autels qui l'avaient restitué dans son nom et glorifié dans sa fonction d'héritier du roi David. Aussi, tandis que son ultime prière, propitiatoire ${ }^{52}$, se révélera vaine (comme le savent spectateurs et lecteurs chrétiens de Racine), entérinant ainsi l'efficace de la parole sacrée, le dernier vers de Britannicus («Plût aux Dieux que ce fût le dernier de ses crimes») doit se lire pour le même public moins comme un souhait tourné vers l'avenir que comme l'expression d'une éventualité encore récusable, voire un regret anticipé dans l'ordre de la Fable. À la clairvoyance d'Agrippine, dont l'ultime réplique de Burrhus ne prend que provisoirement acte, répond la terrible certitude d'Athalie face à un Dieu vengeur dont elle vient d'éprouver la puissance et qu'elle appelle désormais de ses vœux.

L'Histoire profane de Néron, bornée après le cycle de ses crimes à venir et saisie sous le regard des historiens de l'antiquité et de l'âge classique, livre un exemplum à méditer pour les Princes d'ici-bas ${ }^{53}$. Racine en propose une lecture complexe, dès lors que le premier de ses forfaits n'a pas encore été perpétré à l'heure où le rideau s'ouvre. Qu'eût-il fallu faire, ou éviter ? telle est la première leçon morale proposée en guise de "Miroir du Prince ». Mais, représentant de la race humaine corrompue dès l'origine, Néron pouvait-il, sous le regard chrétien venant a posteriori, échapper à un destin que sa mère lui prédit, et que l'Histoire a attesté voire providentiellement justifié ? Dans Athalie en revanche, le triomphe divin de la lignée de David, proclamé par l'héroïne éponyme mais aussitôt rendu à la malédiction originelle contre le sang d'Achab, ne prend sens qu'en second temps, dans le cadre d'une lecture figurative de l'Ancien Testament. Si «David triomphe », ce n'est pas à court terme, puisque Joas apostat reniera son vrai Dieu. L'exclamation d'Athalie, dont l'interprétation lui échappe, mais dont les termes annoncent la véritable leçon sous le voile du sens littéral, préfigure l'avènement du Messie, le Nouveau Testament parachevant les promesses de l'Ancien. 
«Tous chantent de David le Fils ressuscité » (v. 1765), sans comprendre qu'au delà de Joas, c'est bien du Dieu-Père incarné sous l'espèce du Fils qu'il s'agit. Dans le solennel avertissement du grand-prêtre Joad, qui termine la pièce ${ }^{54}$, la leçon eschatologique délivrée doit alors s'entendre non seulement pour le nouveau « Roi des Juifs » qu'est devenu Joas, mais encore à l'égard des puissants de ce monde, comme une prédication véritable, quoique représentée sous fiction dramatique.

Ces imprécations raciniennes vont donc bien au delà de leur seule valeur pathétique, qui confirme par ailleurs l'indéniable efficacité dramatique de cette notion d'origine rhétorique. Leur caractère mémorable, appelant citation, invite à ouvrir l'ordre des temps futurs: ce sublime testamentaire en appelle ainsi à une lecture par paliers, susceptible de déboucher sur un ajustement du regard, ou pour mieux dire, d'en opérer la conversion.

\section{ANNEXES}

\section{Annexe 1. Britannicus, acte V, scène 7, v. 1672-1713}

\section{Agrippine}

Poursuis, Néron, avec de tels Ministres.

Par des faits glorieux tu te vas signaler.

Poursuis. Tu n'as pas fait ce pas pour reculer.

Ta main a commencé par le sang de ton Frère.

Je prévois que tes coups viendront jusqu'à ta Mère.

$\mathrm{Tu}$ te fatigueras d'entendre tes forfaits.

Tu voudras t'affranchir du joug de mes bienfaits.

Mais je veux que ma mort te soit même inutile,

$\mathrm{Ne}$ crois pas qu'en mourant je te laisse tranquille.

Rome, ce ciel, ce jour, que tu reçus de moi,

Partout, à tout moment, m'offriront devant toi.

Tes remords te suivront comme autant de furies.

$\mathrm{Tu}$ croiras les calmer par d'autres barbaries.

Ta fureur s'irritant soi-même dans son cours

D'un sang toujours nouveau marquera tous tes jours.

Mais j'espère qu'enfin le Ciel las de tes crimes

Ajoutera ta perte à tant d'autres victimes,

Qu'après t'être couvert de leur sang et du mien,

Tu te verras forcé de répandre le tien,

Et ton nom paraîtra dans la race future

Aux plus cruels Tyrans une cruelle injure. 
Voilà ce que mon cœur se présage de toi.

Adieu, tu peux sortir.

\section{Annexe 2. Athalie, acte V, scène 6, v. 1768-1790}

\section{Athalie}

Dieu des Juifs, tu l'emportes!

Oui, c'est Joas, je cherche en vain à me tromper.

Je reconnais l'endroit où je le fis frapper.

Je vois d'Ochosias et le port, et le geste.

Tout me retrace enfin un sang, que je déteste.

David, David triomphe. Achab seul est détruit.

Impitoyable Dieu, toi seul as tout conduit.

C'est toi, qui me flattant d'une vengeance aisée,

M'as vingt fois en un jour à moi-même opposée,

Tantôt pour un enfant excitant mes remords,

Tantôt m'éblouissant de tes riches trésors

Que j'ai craint de livrer aux flammes, au pillage.

Qu'il règne donc ce Fils, ton soin, et ton ouvrage.

Et que pour signaler son empire nouveau

On lui fasse en mon sein enfoncer le couteau.

Voici ce qu'en mourant lui souhaite sa Mère.

Que dis-je souhaiter? Je me flatte, j'espère,

Qu'indocile à ton joug, fatigué de ta Loi,

Fidèle au sang d'Achab, qu'il a reçu de moi,

Conforme à son Aïeul, à son Père semblable,

On verra de David l'héritier détestable

Abolir tes honneurs, profaner ton Autel,

Et venger Athalie, Achab, et Jézabel.

\section{NOTES}

* Cette étude a été publiée, en version abrégée, dans La Poésie est grammairienne. Hommages à Joëlle Gardes Tamine, Cl. Ber et Fr. Rullier-Theuret dir., Paris, éd. de l'Amandier, 2012.

1. Voir M. Charles, L'Arbre et la Source, Paris, Le Seuil, 1985.

2. Richesource, Le Masque des Orateurs, c'est à dire La Manière de déguiser facilement toute sorte de Discours, Paris, Académie des Orateurs, 1667, p. 4-5. La formule se précise quinze ans plus tard, et conduit l'auteur à risquer la proposition d'une «seconde rhétorique ", propre à « la Conduite spéciale du Critiste ». Tandis que la première, nommée encore "Synthétique, comme qui dirait faiseuse, composeuse \& ajusteuse, [...] enseigne de quelle manière l'Orateur doit joindre toutes les parties ou pièces d'un discours ", la seconde se voit baptisée d'« Analytique " (Les Plaisirs de la lecture aux vives lumières du Camouflet, Paris, à la Renommée, 1681, art. XXIII, p. 85-86). Nous avons modernisé l'orthographe de toutes nos citations. Sur les propositions de Richesource, voir G. Molinié, «Une rhétorique en action : le cas de Richesource (autour de 1670) », dans Rhétorique et discours critiques. Échanges entre langue et métalangues, Paris, Presses de l'E.N.S., 1989, p. 85-90, 
et D. Denis, "La "seconde rhétorique" de Richesource: critique et pédagogie ", XVII ${ }^{e}$ Siècle, $\mathrm{n}^{\circ}$ 236, 2007, p. 473-486.

3. Britannicus, V, 7, v. 1692-1714; Athalie, V, 6, v. 1768-1790. Nous citons dans l'éd. procurée par G. Forestier (Paris, Gallimard, «Bibliothèque de la Pléiade »,1999) : voir ci-dessous, annexes 1 et 2 .

4. Voir notamment P. France, Racine's Rhetoric, Oxford, Clarendon Press, 1965, et les études de G. Declercq.

5. Vie de Jean Racine, s. 1, 1928, p. 110.

6. Handbuch der literarischen Rhetorik. Eine Grundlegung der Literaturwissenschaft, München, M. Hueber, [1960] 1973, 2 vol.

7. Je remercie Francis Goyet et l'équipe RARE qu'il dirige à l'université Stendhal Grenoble 3 de m'avoir facilité l'accès à une partie du corpus concerné.

8. Voir J. Tesmar, Exercitationum rhetoricarum libri VIII, Amsterdam, L. et D. Elzevier, 1657, VII, 13 ;

G. J. Vossius, Rhetorices contractae, sive Partitionum oratoriarum libri V, Leyde, J. Maire, 1640, II, 26 et 27 ; M. Du Cygne, Explanatio rhetoricae, Köln, V J. Widenfelt, 1670, liv. II.

9. P. T. N. Hurtaut, Manuale Rhetorices, Paris, chez l'auteur, 1782, p. 299.

10. Chez P. Melanchthon : voir le Corpus Reformatum, t. XVII, col. 13 à 560.

11. J. L. de La Cerda, Virgilii Maronis Priores sex libri Aeneidos, Lyon, H. Cardon, 1612 ; G. J. Vossius, Rhetorices contractae, op. cit.; <M. A. Ferrazzi>, Exercitationes rhetoricae in praecipuas P. Virgilii Maronis orationes, Padoue, <Manfrè>, 1694.

12. La Patrologie latine de l'abbé J.-P. Migne (1844-1855) abonde de références en la matière.

13. La Poétique de J. Pilet de La Mesnardière (1639-1640) et La Pratique du théâtre de Fr. Hédelin, abbé d'Aubignac (1657).

14. «Car là, Parler c'est Agir »: La Pratique du théâtre, H. Baby éd., Paris, H. Champion, 2001, p. 407. Les italiques sont de l'auteur.

15. La Mesnardière, La Poétique, Paris, A. de Sommaville, 1639-1640, p. 335 (italiques dans le texte) ; «Si la Poésie est l'Empire des Figures, le Théâtre en est le Trône » (La Pratique du théâtre, op. cit., p. 474.)

16. La Mesnardière, La Poétique, op. cit., p. 375.

17. Ibid., p. 374.

18. Ibid., p. 375.

19. Id.

20. La Pratique du théâtre, op. cit., p. 422.

21. Ibid., p. 459.

22. Ibid., p. 467.

23. Elles sont opposées aux figures de mots jugées « de peu de conséquence » et "affecté[e]s par étude " (ibid., p. 471).

24. Ibid., p. 473.

25. Ibid., p. 476-481.

26. Ibid., p. 476.

27. Id. L'abbé d'Aubignac en illustre la réussite moderne avec un exemple emprunté à Corneille.

28. À bien comprendre l'auteur, on constate que ce sont autant d'affirmations ou de fictions de présence qui surenchérissent sur la dialogicité constitutive du langage dramatique.

29. Ibid., p. 481.

30. La Poétique, XIV, $1453 \mathrm{~b}$.

31. Dans Trois discours sur le poème dramatique [1660], B. Louvat et M. Escola éd., Paris, Flammarion, 1999, p. 106 : «C'est donc un grand avantage pour exciter la commisération que la proximité du sang [...] entre le persécutant et le persécuté, le poursuivant et le poursuivi, celui qui fait souffrir et celui qui souffre ». Un peu plus loin, Corneille ajoutait que de tels sujets ne sauraient être inventés à plaisir, tant ils semblent manquer à la vraisemblance : aussi doivent-ils 
être empruntés à l'Histoire ou à la Fable (ibid., p. 112). Voir G. Forestier, Essai de génétique théâtrale. Corneille à l'œuvre, Paris, Klincksieck, 1996, p. 109-111.

32. L'expression littérale figure chez Corneille (Othon, $\mathrm{V}, 6)$, mais Racine en diffracte la formule à de nombreuses reprises dans l'ensemble de son œuvre.

33. Voir M. Serres, Rome. Le livre des fondations, Paris, Grasset, 1983.

34. Genèse, IV, 10 : « La voix du sang de votre frère crie de la terre jusqu'à moi » (trad. Lemaître de Sacy).

35. Voir P.Sellier, Le Siècle de Saint-Augustin, La Rochefoucauld, Mme de Lafayette, Sacy, Racine, Paris, H. Champion, 2000.

36. Britannicus, v. 1673 ; Athalie, v. 1780-1782.

37. « Toujours humble, toujours le timide Néron / N'ose-t-il être Auguste, et César que de nom ?» (Britannicus, I, 2, v.197-198).

38. Pour une synthèse sur la notion, voir C. Kerbrat-Orecchioni, Les Actes de langage dans le discours. Théorie et fonctionnement, Paris, Nathan Université, 2001.

39. Voir Athalie, v. 1783, 1784, et Britannicus, v. 1679, 1676, 1687.

40. Avec le subjonctif de souhait: "Qu'il règne donc ce Fils, ton soin, et ton ouvrage. / Et que pour signaler son empire nouveau/On lui fasse en mon sein enfoncer le couteau » (Athalie, v. 1780-1782). Nos italiques.

41. À la forme positive puis négative : «Mais je veux que ma mort te soit même inutile : / Ne crois pas qu'en mourant je te laisse tranquille " (Britannicus, v. 1679-1680).

42. Le subjonctif présent, par son aspect tensif (non-accompli), laisse ouverte cette possibilité qu'il ne donne pas comme sûre, là où les nombreux futurs de l'indicatif en prennent le pari (Britannicus, v. 1682-1691; «On verra de David l'héritier détestable / Abolir tes honneurs, profaner ton Autel », Athalie, v. 1789), relayés par leurs équivalents périphrastiques («Par des faits glorieux tu te vas signaler », Britannicus, v. 1673).

43. «Partout, à tout moment » (Britannicus, v. 1682), «D'un sang toujours nouveau marquera tous tes jours» (ibid., v. 1686) ; « Tout me retrace enfin un sang, que je déteste» (Athalie, v. 1772), « Toi seul a tout conduit » (ibid., v. 1774).

44. Britannicus, v. 1683 : « autant de furies »; «tant d'autres victimes » (ibid., v. 1688).

45. Britannicus, v. 1692 : « aux plus cruels tyrans »; « vingt fois en un jour à moi-même opposée » (Athalie, v. 1776).

46. Britannicus, v. 1680 : «Ne crois pas qu'en mourant je te laisse tranquille»; Athalie, v. 1783 : «Voici ce qu'en mourant lui souhaite sa Mère ».

47. M. Deguy, «Le Grand-dire. Pour contribuer à une relecture du pseudo-Longin », dans Du sublime, Paris, Belin, [1984] 1988, p. 16-17.

48. « Mais j'espère qu'enfin le ciel las de tes crimes / Ajoutera ta perte à tant d'autres victimes » (Britannicus, v. 1687) ; «Qu'il règne donc, ce Fils, ton soin et ton ouvrage » (Athalie, v. 1780) ; «Voilà ce que mon cœur se présage de toi » (Britannicus, v. 1693); «Voici ce qu'en mourant lui souhaite sa Mère » (Athalie, v. 1783). Nos italiques.

49. Dans Britannicus, c'est la combinaison des déterminants démonstratifs et possessifs avec l'indicatif futur qui assure cet effet de présence propre au sublime; dans Athalie, il est marqué par la succession des épithètes en asyndète (indocile, fatigué, fidèle, conforme, semblable) caractérisant le nom honni de Joas auquel Athalie substitue une périphrase (« de David l'héritier détestable »), et par la construction infinitive sur laquelle s'achèvent les trois derniers vers de la tirade.

50. Ce défi ironique des mères clairvoyantes s'exprime dans Britannicus par le déterminant indéfini (de tels ministres), qui laisse entendre par ce non-dit tout le mépris d'Agrippine et par l'antiphrase («Par des faits glorieux tu vas te signaler »), là où il s'adresse, dans Athalie, à Dieu lui-même («Qu'il règne donc ce Fils, ton soin et ton ouvrage / Et que pour signaler ton empire nouveau... »). 
51. Voir la préface, op. cit., p. 372.

52. Acte V, 7, v. 1797-1800.

53. Voir B. Guion, Du bon usage de l'histoire: histoire, morale et politique à l'âge classique, Paris, H. Champion, 2008.

54. «Par cette fin terrible, et due à ses forfaits, / Apprenez, Roi des Juifs, et n'oubliez jamais, / Que les Rois dans le Ciel ont un Juge sévère, / L'innocence un Vengeur, et l'Orphelin un Père ».

\section{AUTEUR}

\section{DELPHINE DENIS}

Université Paris Sorbonne-Paris IV 